\title{
WECKEN TYPE PROBLEMS FOR SELF-MAPS OF THE KLEIN BOTTLE
}

\author{
D. L. GONÇALVES AND M. R. KELLY
}

Received 6 October 2004; Revised 1 March 2005; Accepted 21 July 2005

We consider various problems regarding roots and coincidence points for maps into the Klein bottle $K$. The root problem where the target is $K$ and the domain is a compact surface with non-positive Euler characteristic is studied. Results similar to those when the target is the torus are obtained. The Wecken property for coincidences from $K$ to $K$ is established, and we also obtain the following 1-parameter result. Families $f_{n}, g: K \rightarrow$ $K$ which are coincidence free but any homotopy between $f_{n}$ and $f_{m}, n \neq m$, creates a coincidence with $g$. This is done for any pair of maps such that the Nielsen coincidence number is zero. Finally, we exhibit one such family where $g$ is the constant map and if we allow for homotopies of $g$, then we can find a coincidence free pair of homotopies.

Copyright (c) 2006 D. L. Gonçalves and M. R. Kelly. This is an open access article distributed under the Creative Commons Attribution License, which permits unrestricted use, distribution, and reproduction in any medium, provided the original work is properly cited.

\section{Introduction}

Given a pair of maps $(f, g): X \rightarrow Y$ denote by $\operatorname{Coin}(f, g)$ the set $\{x \in X \mid f(x)=g(x)\}$. Assume $X$ and $Y$ to be compact manifolds of the same dimension, in which case this set is generically a finite set of points. Now suppose that $\left(f_{1}, g_{1}\right),\left(f_{2}, g_{2}\right)$ are homotopic as a pair of maps, and that \# Coin $\left(f_{1}, g_{1}\right)=\# \operatorname{Coin}\left(f_{2}, g_{2}\right)=\operatorname{MC}\left[f_{1}, g_{1}\right]$, where $\mathrm{MC}[f, g]$ denotes the minimal number of coincidence points occurring among all pairs $\left(f, g^{\prime}\right)$ homotopic to $(f, g)$.

A natural question is the following: Is it possible to find a pair of homotopies, $H$ from $f_{1}$ to $f_{2}$ and $G$ from $g_{1}$ to $g_{2}$, such that \# $\operatorname{Coin}(H(\cdot, t), G(\cdot, t))=\operatorname{MC}\left[f_{1}, g_{1}\right]$ for all $t \in[0,1]$ ? In this paper we will refer to this as the 1-parameter minimal coincidence problem and will often shorten this to the minimal coincidence problem. A variation of the above question is to consider the situation where in one of the two coordinates the same function appears, and the homotopy between them is constant. We refer to this as the restricted minimal coincidence problem. If we specialize the restricted problem to the 
case where $X=Y$, both $g_{1}$ and $g_{2}$ are the identity and the homotopy $G$ remains constant this is called the fixed point problem and has been considered in a number of papers $[7,10,15,16]$. The last partially generalized to coincidence in [14]. If we specialize to the case where both $g_{1}$ and $g_{2}$ are the constant map and the homotopy $G$ remains constant this is called the root problem.

In a previous paper the authors studied the coincidence problem when the target $Y$ is the 2-dimensional torus [13] (and for the most part the domain as well.) The primary feature used was that because the torus has a multiplication the two coincidence problems are equivalent and can be reduced to considering a root problem, where computations are not as difficult.

The purpose of this present paper is to study these minimal problems for the case where the target is the Klein bottle. Here we are able to take advantage of the multiplication on the torus only after passing to a two-fold cover. As a by-product of our calculations we obtain the Wecken property for coincidences of self maps of the Klein bottle. For the fixed point problem this was first established by Brouwer [5].

The results obtained in this paper are organized as follows. In Section 2 we consider the root problem and show that in the root free case we can always construct an infinite family of maps no two of which can be joined by a root free homotopy. Here the domain is an arbitrary surface and this result is analogous to that for the torus in [13]. In Section 4 we show that the root problem has an affirmative solution when both the domain and target are the Klein bottle and the end maps are not root free. The result is given in Theorem 4.2.

The main body of this paper is Section 3, which gives a study of coincidence for self maps of the Klein bottle $K$. We show that the Wecken property holds (Theorem 3.8) and we also consider the restricted minimal problem defined above. We establish in Theorem 3.10 the following: in any homotopy class of pairs of maps which contains a coincidence free pair the existence of an infinite family of coincidence free pairs each having the same second map, but each homotopy between two distinct members of the family which is constant on the second factor must have a coincidence point.

Finally, in Section 5 we consider the relationship between the minimal coincidence problem and the restricted minimal problem. In particular, we show that the root problem and the minimal coincidence problem where the second map is the constant map, are not equivalent. The result is given in Corollary 5.2. The proof relies on the fact that the second map is the constant map, is the leg which remains unchanged in the restricted coincidence problem and the pair of maps is root free. In general the relations between the two problems is not known. For example, we do not know if the minimal coincidence problem and the fixed point problem for maps in the Klein bottle are equivalent.

Lastly, we point out that no results are given on these coincidence problems for pairs of maps which have Nielsen coincidence number different from zero. For the torus one has an affirmative answer [13]. But this seems difficult to extend to the Klein bottle.

\section{Root free maps into the Klein bottle}

In [12, Theorem 2.2] it was shown that in the setting of orientable surfaces one could always construct countable families of root free maps for which no two members of the family can be joined by a root free homotopy. In this section we present an analogous 
result when the target space is the Klein bottle $K$. For this result the only restriction on the domain is that the surface have non-positive Euler characteristic.

Let $y_{0} \in K$ be the base point. Let $F$ be the free group on the two generators $a, b$, and let $B=a^{2} b^{2}$, the relation which defines the fundamental group of the Klein bottle. Given a reduced word $w \in F$ and an integer $n$ let $w(a, n)$ be the word obtained by replacing each $a$ by $B^{n} a$ and each $a^{-1}$ by $a^{-1} B^{-n}$.

Lemma 2.1. Consider a cyclically reduced word $w \in F$. If $w \neq b^{k}$ for some $k$, then $w(a, n)$ and $w(a, m)$ are not conjugate for $m, n$ different positive integers.

Proof. This can be proved in exactly the same manner as was [12, Lemma 2.1].

Lemma 2.2. Let $U$ be a word, not necessarily reduced, in $F$. If $U=1$ as a reduced word, then for any integer $n, U(a, n)=1$.

Proof. Just follow the cancellation pattern for $U$, cancelling $B, B^{-1}$ pairs along the way.

Let $S$ be a closed surface with non-positive Euler characteristic. Let $e_{1}, \ldots, e_{k}$ be generators for the fundamental group $\pi_{1}(S)$ with defining relation given by; $\left[e_{1}, e_{2}\right] \times \cdots \times$ $\left[e_{h-1}, e_{h}\right]=1$ if $F$ is orientable (here $h$ is even) or by $e_{1}^{2} \times \cdots \times e_{h}^{2}=1$ if $F$ is non-orientable $(h \geq 2)$.

Theorem 2.3. Suppose $f_{0}: S \rightarrow K$ is such that $y \notin f_{0}(S)$ for some $y \in K$. Then there exist a countable family of maps $f_{n}$, each homotopic to $f_{0}$, such that $y \notin f_{n}(S)$ and for any two maps $f_{m}, f_{n}$ in the family with $m \neq n$ each homotopy between them has a root at $y$.

Proof. First identify $\pi_{1}(K-y)$ with the free group $F$ generated by $a$ and $b$. Consider the homomorphism $f_{\#}: \pi_{1}(S) \rightarrow \pi_{1}(K)$ induced by $f_{0}$ which we express by $f_{\#}\left(e_{i}\right)=w_{i}$, where each $w_{i}$ is a word in $F$. The defining relation for $S$ implies the equation $\left[w_{1}, w_{2}\right] \times \cdots \times$ $\left[w_{h-1}, w_{h}\right]=1$ when $S$ is orientable or $w_{1}^{2} \times \cdots \times w_{h}^{2}=1$ when $S$ is non-orientable.

In the special case that $w_{i}=1$ for each $i$, and since $h \geq 2$, we define a family of maps by $f_{n}\left(e_{1}\right)=B^{n}, f_{n}\left(e_{2}\right)=B^{-n}$ and $f_{n}\left(e_{i}\right)=1$ for each $i>2$. These are well defined maps from $S$ into $K-y$, and since $B=1$ in $K$, each is homotopic to any other as maps into $K$. On the other hand, when $n \neq m$ the words $B^{n}$ and $B^{m}$ are not conjugate in $F$. Hence, $f_{n}$ and $f_{m}$ are not homotopic as maps into $K-y$.

Now suppose that at least one of the $w_{i}$ 's is non-trivial. Without loss of generality we can assume that one such word $w_{j}$ is cyclically reduced and is not a power of $b$. Define a family of maps by $f_{n}\left(e_{i}\right)=w_{i}(a, n)$, which by Lemma 2.2, is a well defined family of maps into $K-y$. Since $w_{i}(a, n)=w_{i}$ in $K$, each is homotopic to any other member as maps into $K$. But by [12, Lemma 2.1] if $S$ is orientable, or Lemma 2.1 if $S$ is non-orientable, $f_{n}$ and $f_{m}$ are not homotopic as maps into $K-y$ when $n \neq m$ and both are positive.

\section{Coincidence free maps from the Klein bottle into the Klein bottle}

In this section we consider the situation where the domain and target are the Klein bottle and the pair of maps $(f, g)$ is coincidence free. The purpose of the section is to give two 
results regarding such pairs of maps. In the first section we address the Wecken problem for coincidence free pairs. To do so we set up the notation and preliminary results needed for the result, given in Theorem 3.8, that the Wecken property holds for coincidences on the Klein bottle. In the second section we restrict our attention to those homotopies for which one of the two factors is kept constant; either the map $f$ at each level or $g$ at each level. We then consider the restricted 1-parameter problem and obtain the result stated in Theorem 3.10.

3.1. The Wecken property. Fix generators $\alpha, \beta$ for $\pi_{1}\left(K, 1^{\prime}\right)$ so that the relation $\alpha \beta \alpha \beta^{-1}=$ 1 holds. Let $p: T \rightarrow K$ be the double cover by the torus, and let $a, b$ generate $\pi_{1}(T, 1)$, where $p(a)=\alpha$ and $p(b)=\beta^{2}$. As an abuse of notation both $a$ and $b$ will also represent simple closed curves meeting at the basepoint of the torus.

To prove this result we first recall the homotopy classification of self-maps of the Klein bottle.

Lemma 3.1. Let $f: K \rightarrow K$ be given. Then $f_{\#}$ has one of two forms. Either Type $I: f_{\#}(\alpha)=\alpha^{r}$ $f_{\#}(\beta)=\alpha^{s} \beta^{2 q+1}$ or Type II: $f_{\#}(\alpha)=1 f_{\#}(\beta)=\alpha^{s} \beta^{2 q}$.

Proof. The Klein bottle relation $\alpha \beta \alpha \beta^{-1}=1$ viewed as $\alpha \beta=\beta \alpha^{-1}$ allows for any word to be converted to a word of the form $\alpha^{a} \beta^{b}$. In the process the exponent sum on $\beta$ remains the same. As a result, and since $K$ is a $K(\pi, 1)$, all self-maps are represented by a member of the family given by

$$
\alpha \longmapsto \alpha^{r} \beta^{u}, \quad \beta \longmapsto \alpha^{s} \beta^{t}
$$

Apply this map to the Klein bottle relation to get $\alpha^{r} \beta^{u} \alpha^{s} \beta^{t} \alpha^{r} \beta^{u} \beta^{-t} \alpha^{-s}=1$. When applying the relation to put this word in normal form we see that the exponent on $\beta$ is $2 u$. Hence, $u=0$. Also, if $t$ is even, then $\beta^{t} \alpha^{r}=\alpha^{r} \beta^{t}$. The equation above reduces to $\alpha^{2 r}=1$, and so $r=0$.

Remark 3.2. Following classical notation the map $f$ is orientation-true, see [6] or [9], exactly when it is Type I. In those papers all other maps are classified as either Type II or III. To simplify notation in this paper we will not use Type III. We simply note that the only self-map of $K$ that is of Type III is the constant map, which we will consider under the case of Type II.

As a notation we will index maps as a triple $(r, s, t)$, where $r, s$ are as above and $t$ is the $\beta$ exponent of $f_{\#}(\beta)$. When $t$ is even we must have that $r=0$. For a given pair $\left(f_{1}, f_{2}\right)$ of maps there is a formula for the Nielsen coincidence number of the pair.

Theorem 3.3 [8]. Let $f_{1}, f_{2}: K \rightarrow K$ be as above, with $f_{i}=\left(r_{i}, s_{i}, t_{i}\right)$. Then

$$
N\left(f_{1}, f_{2}\right)=\left|t_{1}-t_{2}\right| \max \left\{\left|r_{1}\right|,\left|r_{2}\right|\right\}
$$

For various calculations in this paper we will be considering lifts of maps to the torus. We list here for reference the form of these lifts. 
In the case that $f$ is Type I we can lift the map by lifting both the domain and the range. Here we get $\tilde{f}: T \rightarrow T$, and with the notation above, $\tilde{f}(a)=a^{r}, \tilde{f}(b)=b^{2 q+1}$. Let $\theta: T \rightarrow$ $T$ denote the deck transformation corresponding to the cover $p$. Then the associated lift $\tilde{f}^{\prime}=\theta \tilde{f}$ satisfies $\tilde{f}^{\prime}(a)=a^{-r}, \tilde{f}^{\prime}(b)=b^{2 q+1}$, where $a, b$ in the target mean the translated loops based at $\theta(1)$.

When $f$ is not orientation-true it factors through the torus. So we have a map $\bar{f}: K \rightarrow$ $T$ where $\bar{f}(\alpha)=1$ and $\bar{f}(\beta)=a^{s} b^{q}$. In this case the associated lift $\overline{f^{\prime}}$ is $\overline{f^{\prime}}(\alpha)=1$ and $\overline{f^{\prime}}(\beta)=a^{-s} b^{q}$. We can also lift in the domain as well. When we do so we now have that $\tilde{f}$ is given by $\tilde{f}(a)=1$ and $\tilde{f}(b)=a^{2 s} b^{2 q}$, and that $\widetilde{f^{\prime}}(a)=1$ and $\widetilde{f^{\prime}}(b)=a^{-2 s} b^{2 q}$. As before, for $\tilde{f}$ and $\tilde{f}^{\prime}$ the loops in the target are based at $\theta(1)$.

At this point we digress a moment to compare a result for maps on the torus to its counterpart for the Klein bottle. A class of spaces called Jiang type spaces, which includes the torus, has the property that when the Nielsen number of a pair of maps is zero then the Reidemeister number is infinite, and when the Nielsen number is nonzero the two are equal (see, e.g., [19].) The next proposition shows that this does not hold for the Klein bottle, even in the fixed point case.

Proposition 3.4. Let $f_{1}, f_{2}: K \rightarrow K$ be as above, with $f_{i}=\left(r_{i}, s_{i}, t_{i}\right)$. Then the coincidence Reidemeister number for the pair is infinite exactly when either $N\left(f_{1}, f_{2}\right)=0$ or $\left|r_{1}\right|=\left|r_{2}\right|$.

Proof. This follows directly from the matrices which arise when lifting $f_{1}, f_{2}$ to maps of the torus.

In order to study coincidences of pairs of maps on the Klein bottle we will first lift maps to the torus and then use a multiplication on the torus to reduce to the root problem for a deviation map $h$ given by $h(x)=\tilde{f}_{1}(x) \tilde{f}_{2}(x)^{-1}$. We now vary $h$ by a homotopy for the root problem, and then recover equivariant maps on the torus by keeping $\tilde{f}_{2}$ fixed and obtaining a new $\tilde{f}_{1}$ from this formula.

As an immediate consequence of the definition of $h$ and Theorem 3.3 we have the following lemma. Its proof is left to the reader.

Lemma 3.5. If $N\left(f_{1}, f_{2}\right)=0$, then the map $h: T \rightarrow T$ has the property that $a \mapsto a^{r_{1}-r_{2}}$, where $r_{i}=0$ when $f_{i}$ is of Type II. Furthermore, $r_{1}-r_{2}$ is non-zero only when both maps are of Type I and $t_{1}=t_{2}$.

As a consequence we see that the Wecken problem for coincidence in the case $N\left(f_{1}, f_{2}\right)$ $=0$ and $r_{1}=r_{2}$ is now easy to solve. Since the loop $a$ is mapped by $h$ to a single point, $h(\theta(a))$ must also be a single point. As a result $h$ maps $T$ into a 1-complex determined by $h(b)$. After a small deformation we arrange that $h$ is a root free map at both 1 and $\theta(1)$. Thus, both pairs of lifts $h \tilde{f}_{2}, \tilde{f}_{2}$ and $h \tilde{f}_{2}, \theta \tilde{f}_{2}$ are coincidence free, and we conclude that $\left(f_{1}, f_{2}\right)$ can be deformed to a coincidence free pair. Details are given in the proof of Proposition 3.4.

The case when $r_{1} \neq r_{2}$ is more subtle. To analyze this case, and also to deal with the 1parameter problem, we will need to see how the various lifts, and hence $h$, act on a certain 1-complex in $T$. To define this complex let $T$ be the identification of the unit square $[0,1]^{2}$ with $a=\{0\} \times[0,1]$ and $b=[0,1] \times\{0\}$. Let $\sigma_{a}=a$ and $\sigma_{b}=[0,1 / 2] \times\{0\}$. Our 
1-complex $L$ is the union of $a, b$ and $\theta(a)$. The action of an equivariant map is determined by the action on $\sigma_{a}$ and on $\sigma_{b}$.

Given $f: K \rightarrow K$ which is assumed to be given efficiently in terms of the generators $\alpha, \beta$, a model for a lift of $f$ on $\sigma_{a} \cup \sigma_{b}$ is given by the following lemma

Lemma 3.6. If $f$ is Type I, then $\tilde{f}\left(\sigma_{a}\right)=a^{r}, \tilde{f}\left(\sigma_{b}\right)=a^{s} b^{q} \sigma_{b}$. If $f$ is Type II, then

$$
\tilde{f}\left(\sigma_{a}\right)=1, \quad \tilde{f}\left(\sigma_{b}\right)=a^{s} b^{q}
$$

We now check the action of $h: T \rightarrow T$ on $L$ by first computing $h(\theta z)=\tilde{f}_{1}(\theta z)\left(\tilde{f}_{2}(\theta z)\right)^{-1}$. We express points in complex $(x, y)$ coordinates where $x$ measures the $b$ or $\beta$ direction, and $y$ the $a$ or $\alpha$ direction, depending on location in $T$ or in $K$. The action of $\theta$ is given by $(x, y) \mapsto\left(e^{\pi i} x, \bar{y}\right)$, and multiplication is the product of the coordinates. The inverse is obtained by complex conjugation in each coordinate.

One feature of multiplication that we exploit is its relation with the deck transformation $\theta$. Namely, under products we see that

$$
\theta(A B)=\theta(A) \theta(B)\left(e^{\pi i}, 1\right) .
$$

Case 1. Both $f_{1}, f_{2}$ are Type I. ( $t_{1}, t_{2}$ odd.)

Then reduce $h(\theta z)$ to $\theta \tilde{f}_{1}(z)\left(\theta \tilde{f}_{2}(z)\right)^{-1}$. This is equal to $\theta\left(h(z) \tilde{f}_{2}(z)\right)\left(\theta \tilde{f}_{2}(z)\right)^{-1}$, or $\theta h(z) \theta \tilde{f}_{2}(z)\left(e^{\pi i}, 1\right)\left(\theta \tilde{f}_{2}(z)\right)^{-1}=\theta h(z)\left(e^{\pi i}, 1\right)$.

Case 2. Both $f_{1}, f_{2}$ are Type II. $\left(t_{1}, t_{2}\right.$ even.)

Then reduce to $\tilde{f}_{1}(z)\left(\tilde{f}_{2}(z)\right)^{-1}=\left(h(z) \tilde{f}_{2}(z)\right)\left(\tilde{f}_{2}(z)\right)^{-1}=h(z)$.

Case 3. $t_{1}$ odd, $t_{2}$ even.

Reduce to $\theta\left(h(z) \tilde{f}_{2}(z)\right)\left(\tilde{f}_{2}(z)\right)^{-1}$. Which is $\theta h(z) \theta \tilde{f}_{2}(z)\left(e^{\pi i}, 1\right)\left(\tilde{f}_{2}(z)\right)^{-1}$.

Using coordinates for $\tilde{f}_{2}(z)=\left(u_{b}, u_{a}\right)$, this reduces to $\theta h(z)\left(1, u_{a}^{-2}\right)$.

Case 4. $t_{1}$ even, $t_{2}$ odd.

Reduce to $\left(h(z) \tilde{f}_{2}(z)\right)\left(\theta \tilde{f}_{2}(z)\right)^{-1}$ which is $h(z)\left(e^{\pi i}, u_{a}^{2}\right)$.

We now revisit these four cases using the given information of $h(z)$ on the loop $\sigma_{a}$ and the path $\sigma_{b}$ as given in Lemma 3.6.

Case 1. In view of Lemma 3.5 we divide this case into two subcases.

Subcase $1(i) . \quad r_{1} \neq r_{2}$. Here $h\left(\sigma_{a}\right)=\left(1, a^{r_{1}-r_{2}}\right)$, and since $q_{1}=q_{2}, h\left(\sigma_{b}\right)=\left(1, a^{s_{1}-s_{2}}\right)$.

So we get $h\left(\theta \sigma_{a}\right)=\theta\left(1, a^{r_{1}-r_{2}}\right)\left(e^{\pi i}, 1\right)=\left(1, a^{r_{2}-r_{1}}\right)$, and similarly, $h\left(\theta \sigma_{b}\right)=\theta\left(1, a^{s_{1}-s_{2}}\right)$ $\times\left(e^{\pi i}, 1\right)=\left(1, a^{s_{2}-s_{1}}\right)$.

We see from these equations that on the complex $L$ the action of $h$ is by a power of $a$. Moreover, consider the loop in $L$ formed by (in order) $\sigma_{a},[0,1 / 2] \times\{1\},\{1 / 2\} \times$ $[1,0], \sigma_{b}^{-1}$. (Or equivalently $\sigma_{a} \sigma_{b} \theta\left(\sigma_{a}\right) \sigma_{b}^{-1}$.) The action of $h$ on this loop is given by the word $h\left(\sigma_{a}\right) h\left(\sigma_{b}\right) h\left(\theta \sigma_{a}\right) h\left(\sigma_{b}\right)^{-1}$. But this is $\left(1, a^{r_{1}-r_{2}}\right)\left(1, a^{s_{1}-s_{2}}\right)\left(1, a^{r_{2}-r_{1}}\right)\left(1, a^{s_{2}-s_{1}}\right)=(1,1)$. A similar calculation on the other loop in $L$ shows that the exponents cancel to zero as well. As a result we can arrange that $h(T)$ is contained in a 1-complex determined by $h\left(\sigma_{a}\right)$ 
and so this representative gives us a root free map. Moreover, keeping $\tilde{f}_{2}$ fixed, from the construction we see that the corresponding $\tilde{f}_{1}$ is an equivariant map. So we have a coincidence free pair.

Subcase 1(ii). $r_{1}=r_{2}$. Then $\sigma_{a} \mapsto 1, \sigma_{b} \mapsto\left(b^{q_{1}-q_{2}}, a^{s_{1}-s_{2}}\right)$ by $h$. So $h\left(\theta \sigma_{a}\right)=\theta(1,1)\left(e^{\pi i}, 1\right)=$ $(1,1)$ and $h\left(\theta \sigma_{b}\right)=\theta\left(b^{q_{1}-q_{2}}, a^{s_{1}-s_{2}}\right)\left(e^{\pi i}, 1\right)=\left(b^{q_{1}-q_{2}}, a^{s_{2}-s_{1}}\right)$.

Here $h$ as defined on $L$ extends to $T$ by mapping into a 1-complex, this time determined by $h\left(\sigma_{b}\right)$. This will also happen in the remaining three cases.

Case 2. Here $a \mapsto 1, \sigma_{b} \mapsto\left(b^{q_{1}-q_{2}}, a^{s_{1}-s_{2}}\right) h\left(\theta \sigma_{a}\right)=h\left(\sigma_{a}\right)(1,1)=(1,1)$ and $h\left(\theta \sigma_{b}\right)=h\left(\sigma_{b}\right)(1$, $1)=\left(b^{q_{1}-q_{2}}, a^{s_{1}-s_{2}}\right)$.

Case 3. $a \mapsto 1, \sigma_{b} \mapsto\left(b^{q_{1}-q_{2}} \sigma_{b}, a^{s_{1}-s_{2}}\right) h\left(\theta \sigma_{a}\right)=\theta(1,1)\left(1, u_{a}^{-} 2\right)=\left(e^{\pi i}, 1\right)$, as $\left(u_{b}, u_{a}\right)=(1,1)$ in this case, and $h\left(\theta \sigma_{b}\right)=\theta\left(\left(b^{q_{1}-q_{2}} \sigma_{b}, a^{s_{1}-s_{2}}\right)\left(1, a^{-2 s_{2}}\right)=\left(e^{\pi i} b^{q_{1}-q_{2}} \sigma_{b}, a^{-s_{1}-s_{2}}\right)\right.$.

Case 4. $a \mapsto 1, \sigma_{b} \mapsto\left(b^{q_{1}-q_{2}} \sigma_{b}^{-1} a^{s_{1}-s_{2}}\right) h\left(\theta \sigma_{a}\right)=\left(h\left(\sigma_{a}\right)(1,1)\right)\left(e^{\pi i}, 1\right)=\left(e^{\pi i}, 1\right)$ and $h\left(\theta \sigma_{b}\right)$ $=\left(b^{q_{1}-q_{2}} \sigma_{b}^{-1}, a^{s_{1}-s_{2}}\right)\left(e^{\pi i}, a^{2 s_{2}}\right)=\left(e^{\pi i} b^{q_{1}-q_{2}} \sigma_{b}^{-1}, a^{s_{1}+s_{2}}\right)$.

Each of the above calculations can be repeated with $\tilde{f}_{2}$ replaced by $\theta \tilde{f}_{2}$. For example, in the Case 1(i) it can be shown that $h\left(\sigma_{a}\right)=\left(e^{\pi i}, a^{r_{1}+r_{2}}\right), h\left(\sigma_{b}\right)=\left(e^{\pi i}, a^{-s_{1}+s_{2}}\right), h\left(\theta \sigma_{a}\right)=$ $\left(e^{\pi i}, a^{-r_{1}-r_{2}}\right)$, and $h\left(\theta \sigma_{b}\right)=\left(e^{\pi i}, a^{s_{1}-s_{2}}\right)$. Similar formulas arise in all the other cases.

As a result, we see that the deviation map $h$ has image in a 1-complex. In particular, the image could be taken to be in the "lines" in the torus determined by $h(b)$. As a consequence we can now show that the Klein bottle has the Wecken property for coincidences.

Proposition 3.7. Let $f_{1}, f_{2}: K \rightarrow K$ be such that $N\left(f_{1}, f_{2}\right)=0$. Then we can deform the pair to one that is coincidence free.

Proof. Choose representatives and lifts $\tilde{f}_{1}, \tilde{f}_{2}$ as above. Let $y$ be a point on the torus. Consider the map $h_{y}: T \rightarrow T$ constructed just as $h$, but now given on $L$ by the data $\sigma_{a} \mapsto y h\left(\sigma_{a}\right), \sigma_{b} \mapsto y h\left(\sigma_{b}\right)$. In all cases the image of $h_{y}$ lies in a 1-complex, and for a suitable choice of $y$ the image lies in $T-(1 \cup \theta(1))$.

Now, keeping $\tilde{f}_{2}$ fixed we construct from $h_{y}$ an equivariant map $\tilde{f}_{1}^{\prime}$ such that each of the pairs $\left(\tilde{f}_{1}^{\prime}, \tilde{f}_{2}\right)$ and $\left(\tilde{f}_{1}^{\prime}, \theta\left(\tilde{f}_{2}\right)\right)$ is coincidence free. Hence, $\left(f_{1}^{\prime}, f_{2}\right)$ is coincidence free where $f_{1}^{\prime}$ is the projection of $\tilde{f}_{1}^{\prime}$.

TheOREM 3.8. Given any pair $f_{1}, f_{2}: K \rightarrow K$ we can deform the pair to one that has exactly $N\left(f_{1}, f_{2}\right)$ coincidence points.

Proof. When the Nielsen number is nonzero we show that the "linear" model has exactly $N\left(f_{1}, f_{2}\right)$ coincidence points. This model is obtained by lifting to the torus where we have a piecewise linear map. We present the case where $f_{1}$ is of Type I and $f_{2}$ is of Type II. The details of the other cases are similar and left to the reader.

By Lemma 3.6 we have that $\widetilde{f}_{1}\left(\sigma_{a}\right)=a^{r_{1}}, \tilde{f}_{1}\left(\sigma_{b}\right)=a^{s_{1}} b^{q_{1}} \sigma_{b}, \tilde{f}_{2}\left(\sigma_{a}\right)=1, \tilde{f}_{2}\left(\sigma_{b}\right)=a^{s_{2}} b^{q_{2}}$. The map $\tilde{f}_{2}$ extends to a linear map of the torus given by $(x, y) \mapsto\left(2 q_{2} x, 2 s_{2} x\right)(\bmod 1)$, where the $x$-factor corresponds to the $b$ direction. The map $\tilde{f}_{1}$ can be represented by the map $(x, y) \mapsto\left(\left(2 q_{1}+1\right) x, r_{1} y \pm s_{1} x\right)(\bmod 1)$. The choice of \pm depends on the value $x,+$ if $0<x<0.5,-$ if $0.5<x<1$. Finding coincidence points for the pair $\left(\tilde{f}_{1}, \tilde{f}_{2}\right)$ reduces 
to solving the equations $\left(2 q_{1}+1-2 q_{2}\right) x=0(\bmod 1)$ and $\left(r_{1} y \pm s_{1} x\right)-2 s_{2} x=0(\bmod 1)$. The first has exactly $\left|\left(2 q_{1}+1-2 q_{2}\right)\right|$ solutions and for each of these solutions the second equation has $\left|r_{1}\right|$ solution provided that $r_{1} \neq 0$.

In the case under consideration the coincidence set for the lifts projects one-to-one and onto the coincidence set for $\left(f_{1}, f_{2}\right)$ and so by Theorem 3.3 the result is proved.

3.2. The 1-parameter problem. For the problem of deforming a pair of maps to one that has the least number of coincidence points it is known, in the setting of closed manifolds, that it suffices to deform either one of the two maps [2]. For the 1-parameter Wecken problem this is not known in general, but does hold when the target is a topological group.

The following proposition shows that when considering the restricted coincidence problem (where the second factor $g$ is unchanged) the solution does not depend on the choice of map in the homotopy class.

Proposition 3.9. Let $(f, g): M \rightarrow N$ be a pair of maps which satisfies $\operatorname{Coin}(f, g)=M C(f$, $g)$ and $g_{1}$ a map homotopic to $g$. Then:

(a) the restricted minimal coincidence problem has a positive solution for $(f, g)$ if and only if it has a solution for a pair $\left(f^{\prime}, g\right)$, where $f^{\prime}$ is homotopic to $f$;

(b) there exists $f_{1}$ homotopic to $f$ such that $\operatorname{coin}\left(f_{1}, g_{1}\right)=M C(f, g)$ and the restricted minimal coincidence problem has a positive solution for $(f, g)$ if and only if it has a solution for the pair $\left(f_{1}, g_{1}\right)$.

Proof. In both parts (a) and (b) it suffices to assume that the Wecken problem has a positive solution. For the part (a) we have that the pair $(f, g)$ can be connected to the pair $\left(f^{\prime}, g\right)$ by a Wecken homotopy. So given any pair $\left(f^{\prime \prime}, g\right)$ where $f^{\prime \prime}$ is homotopic to $f^{\prime}$ (so homotopic to $f$ ) we consider the homotopy which is the Wecken homotopy from $\left(f^{\prime}, g\right)$ to $(f, g)$ followed by a Wecken homotopy from $(f, g)$ to $\left(f^{\prime \prime}, g\right)$, which exists by hypothesis, and the result follows.

For part (b) consider the fibre pair $(N \times N, N \times N-\Delta) \rightarrow N$, by projection on the second coordinate. We argue as in [11, Proposition 1.5], let $H: M \times I \rightarrow N$ be a homotopy between $g$ and $g^{\prime}$. This homotopy restricted to $M \times\{0\}$ has a lift given by $(f, g)$, which maps $M-\operatorname{coin}(f, g)$ into $N \times N-\Delta$. Define $f_{1}$ as the first coordinate function of the map $\tilde{H}$ restricted to $M \times\{1\}$, where $\tilde{H}$ is the lifting of $H$ given by the homotopy property of the pair. So $\tilde{H}(M-\operatorname{coin}(f, g) \times\{t\}) \subset N \times N-\Delta$ for all $t \in[0,1]$ and $f_{1}, g_{1}$ is a minimal pair. Now let $f_{1}^{\prime}$ be any map homotopic to $f_{1}$ (so homotopic to $f$ ) such that the pair $\left(f_{1}^{\prime}, g\right.$ ) is minimal. By using the procedure above we can produce a map $f^{\prime}$ which is homotopic to $f$ and the pair $\left(f^{\prime}, g\right)$ is minimal. So by hypothesis there exists a Wecken homotopy $L$ connecting $(f, g)$ to $\left(f^{\prime}, g\right)$. Now we use the procedure above to produce a Wecken homotopy connecting $\left(f_{1}^{\prime}, g_{1}\right)$ to $\left(f_{1}, g_{1}\right)$. Define $L_{1}: M \times I \times I \rightarrow N$ given by $L_{1}(x, s, t)=$ $H(x, s)$ where $H$ is the homotopy between $g$ and $g^{\prime}$. This map restricted to $M \times\{0\} \times I$ admits a lift given by $L$. Define $L_{1}$ as the first coordinate function of the lift $\tilde{L}_{1}$ of $L_{1}$ restricted to $M \times\{1\} \times I$. This is a Wecken homotopy connecting $\left(f_{1}, g_{1}\right)$ to $\left(f_{1}^{\prime}, g_{1}\right)$ and the result follows.

The main purpose of this section is to prove the following theorem. 
THEOREM 3.10. Let $([f],[g])$ be a pair of homotopy classes of self-maps of $K$ such that $N(f, g)=0$. Then given a map $g^{\prime} \in[g]$ there is a countable family of maps $f_{n}$, where each $f_{n} \in[f]$ and $\operatorname{coin}\left(f_{n}, g^{\prime}\right)=\varnothing$, such that for any two pairs $\left(f_{m}, g^{\prime}\right),\left(f_{n}, g^{\prime}\right)$ with $m \neq n$ there is no homotopy $H$ between $f_{m}, f_{n}$ with the property that $\left(H(, t), g^{\prime}\right)$ is coincidence free for all $t \in[0,1]$.

In order to proceed with the proof of this theorem we will first need to construct a suitable family of maps. In view of Proposition 3.9 we choose $g^{\prime}$ as the linear model determined by the images of $\sigma_{a}$ and $\sigma_{b}$. For the following proof we will use $\tilde{f}_{2}$ to denote the lift of $g^{\prime}$ to the torus. Families in the homotopy class of $[f]$ will constructed for each case, by first defining the maps on $\sigma_{a} \cup \sigma_{b}$, and then using the formulas of Cases 1-4 we define families of deviation maps $h_{l}$. We set $\tilde{f}_{1}(x)=h_{l}(x) \tilde{f}_{2}(x)$ keeping in mind that $\tilde{f}_{1}$ represents an arbitrary member of some family of maps. Finally, we project to get families of maps on $K$. Choices will be made so that $N\left(f_{1}, f_{2}\right)=0$, for each possible $f_{1}$. To get coincidence free pairs we will need to make a slight perturbation, which will be done on the torus.

We first consider the situation in Case 1. If a segment of $\sigma_{a} \cup \sigma_{b}$ is mapped by $h$ to the loop $a$, then $h \theta$ of the segment is mapped to $(\theta a)\left(e^{\pi i}, 1\right)$. But this is just the loop $a^{-1}$. Similarly, if a segment of $\sigma_{a} \cup \sigma_{b}$ traces out the loop $b$, then $h \theta$ of the segment is mapped to $b$. As a result, under the hypothesis of Case 1 we have the following method of substitution.

Lemma 3.11. Suppose that both $f_{1}$ and $f_{2}$ are of Type I. Let $W$ be a word in the letters $a, b$ and let $\bar{W}$ be the word obtained by replacing each occurrence of a with $a^{-1}$ keeping $b$ with unchanged. If $h\left(\sigma_{l}\right), l \in\{a, b\}$, contains $W$, then $h\left(\theta \sigma_{l}\right)$ contains $\bar{W}$ in its place.

We now define families of pairs of maps in a given homotopy class of pairs. We first make an adjustment so that all maps are coincidence free.

Let $\epsilon: T \rightarrow T$ be a homeomorphism near the identity and such that $\epsilon(a)$ and $\epsilon(b)$ do not contain 1 . Our deviation maps will be defined on generators $a, b$ and will have images in $\epsilon(a) \cup \epsilon(b)$, and will extend to the interior of $T$ with image in the same 1-complex. Clearly, any such map will have no root at 1 . By abuse of notation in the following we will write $a, b$ instead of $\epsilon(a), \epsilon(b)$. For instance, $h(a)=a b$ means the image of $a$ traces out $\epsilon(a)$ then $\epsilon(b)$.

Let $B$ denote the commutator $a b a^{-1} b^{-1}$ in the free group on generators $a, b$. Given a word $\omega$, let $B_{\omega}=\omega B \omega^{-1}$.

Let $h$ be as presented in Case 1. For integers $m, x, y$ we define a family of maps $h_{m, x, y}$ according to the following:

$$
\sigma_{a} \longmapsto B_{a^{x}}^{m} B_{a^{y}}^{m} a^{r_{1}-r_{2}}, \quad \sigma_{b} \longmapsto a^{s_{1}-s_{2}} .
$$

Keeping $\tilde{f}_{2}$ fixed each $h_{m, x, y}$ determines a family of lifts $\tilde{f}_{1}$, where each pair is homoptic to the original model.

The following gives a condition that ensures our pair is coincidence free. 
LemmA 3.12. If $x+y=r_{1}-r_{2}+s_{1}-s_{2}-1$, then $h_{m, x, y}$ extends to all of $T$ with image in $a \cup b$.

Proof. We check the condition for cancellation around the loop $\sigma_{a} \sigma_{b}\left(\theta \sigma_{a}\right) \sigma_{b}^{-1}$. The other loop in $L$ is the same. Set $r=r_{1}-r_{2}$ and $s=s_{1}-s_{2}$. Then this is equivalent to the equation

$$
B_{a^{x}}^{m} B_{a^{y}}^{m} a^{r}=a^{s}\left(\overline{B_{a^{x}}^{m} B_{a^{y}}^{m} a^{r}}\right)^{-1} a^{-s}=a^{s} a^{r} \bar{B}_{a^{-y}}^{-m} \bar{B}_{a^{-x}}^{-m} a^{-s}=a^{s+r} B_{a^{-1-y}}^{m} B_{a^{-1-x}}^{m} a^{-s},
$$

due to the fact that $\bar{B}^{-1}=B_{a^{-1}}$. Rewrite the right-hand side as

$$
B_{a^{r+s-1-y}}^{m} B_{a^{r+s-1-x}}^{m} a^{r+s} a^{-s}
$$

Equate with the left-hand side to obtain $x+y=r+s-1$ which gives the desired result.

\section{Proof of Theorem 3.10}

Case 1(i). Suppose two pairs from the construction above which also satisfy the conclusion of Lemma 3.12 are joined by a coincidence free homotopy. Lift the homotopy to the torus to get a coincidence free homotopy between the corresponding pairs. Choose the lift so that it produces the deviation map of the form $\sigma_{a} \mapsto a^{r}, \sigma_{b} \mapsto a^{s}$.

As a result we have a homotopy $h_{t}$ between two deviation maps $h_{0}=h_{m_{1}, x_{1}, y_{1}}$ and $h_{1}=$ $h_{m_{2}, x_{2}, y_{2}}$ with no root at 1 for each level of the homotopy. Hence, $h_{0}$ and $h_{1}$ are homotopic as maps into $T-1$ (recall that $a, b$ below are actually $\epsilon(a), \epsilon(b)$.) Since $\sigma_{a}$ is a loop its image under each level of the homotopy is a loop in $T-1$. This implies that there exists a word $\phi$ in the free group such that

$$
B_{a^{x_{1}}}^{m_{1}} B_{a^{y_{1}}}^{m_{1}} a^{r}=\phi\left(B_{a^{x_{2}}}^{m_{2}} B_{a^{y_{2}}}^{m_{2}} a^{r}\right) \phi^{-1}
$$

or

$$
\begin{gathered}
B_{a^{x_{1}}}^{m_{1}} B_{a^{y_{1}}}^{m_{1}} B_{a^{y_{2}}}^{-m_{2}} B_{a^{x_{2}}}^{-m_{2}}=\phi\left(B_{a^{x_{2}}}^{m_{2}} B_{a^{y_{2}}}^{m_{2}} a^{r}\right) \phi^{-1} a^{-r}\left(B_{a^{x_{2}}}^{m_{2}} B_{a^{y_{2}}}^{m_{2}}\right)^{-1}, \\
B_{a^{x_{1}}}^{m_{1}} B_{a^{y_{1}}}^{m_{1}} B_{a^{y_{2}}}^{-m_{2}} B_{a^{x_{2}}}^{-m_{2}}=\left[\phi,\left(B_{a^{x_{2}}}^{m_{2}} B_{a^{y_{2}}}^{m_{2}} a^{r}\right)\right] .
\end{gathered}
$$

So by [1, Theorem 9.1 part (b)], this equation has no solution if $2\left|m_{1}-m_{2}\right|>4$ (with $h=g=1$ and $l=4$.) Therefore if we take the sequence of integers of the form $3 n$ we obtain the result.

Case 1(ii). In order to facilitate computations we take as our base pair $\left(f_{1}, f_{2}\right)$ obtained from the deviation map given by $\sigma_{a} \mapsto 1 \sigma_{b} \mapsto X$, where $X$ is the path in $a \cup b$ corresponding to the word $a^{k} b^{l}$, where $k=s_{1}-s_{2}$ and $l=q_{1}-q_{2}$. This pair is homotopic to that given by the deviation map which sends $\sigma_{b}$ to the path $\left(b^{q_{1}-q_{2}}, a^{s_{1}-s_{2}}\right)$. Our family of maps $h_{n}: T \rightarrow T$ given by $\sigma_{a} \mapsto 1, \sigma_{b} \mapsto B^{n} X$ determine equivariant pairs $\tilde{f}_{1}, \tilde{f}_{2}$ as in Case $1(\mathrm{i})$, 
and thus pairs of maps on the Klein bottle. The construction ensures that $h_{n}$ and $h_{m}$ determine the same homotopy class of pairs on $K$. Also, since each maps $\sigma_{a}$ to 1 each $h_{n}$ maps into $T-1$ resulting in coincidence free pairs.

The existence of a coincidence free homotopy between any two pairs lifts and multiplies to a root free at 1 homotopy between $h_{n}$ and $h_{m}$. This implies that $B^{n} X \overline{B^{n} X}=$ $\phi B^{m} X \overline{B^{m} X} \phi^{-1}$. We claim that this only happens when $n=m$.

Given a word $\alpha$ (in the free group on letters $a, b$ ) define the integer $t(\alpha)$ to be the minimal number of transitions between the letters $a^{ \pm}$and $b^{ \pm}$among all words conjugate to $\alpha$. For example, $t\left(a b^{3} a^{-1} b a b^{-1} a\right)=5$. Clearly, $t()$ is an invariant of a conjugacy class.

For the words of the form $B^{n} X\left(\overline{B^{n} X}\right)$ the calculation of $t()$ is straightforward. Using Lemma 3.11 one can show that $t\left(B^{n} X\left(\overline{B^{n} X}\right)\right)=8 n-c$, where $c$ is a constant which depends on $k, l$ and takes values in $\{-3,1,5\}$, with one exception. When $k=1, l=0$ we get $t\left(B^{n} a\left(\overline{B^{n} a}\right)\right)=0$, which is a result of the fact that $\bar{B}=a^{-1} B^{-1} a$.

Hence we are finished with the proof except when $k=1, l=0$. Now to handle this one special case we go back to a different choice.

Of a family of maps. In place of using $B=a b a^{-1} b^{-1}$ we use $\widehat{B}=b a^{-1} b^{-1} a$. Following through the exact same proof, and now with this change the value of $t($ ) becomes $8 n-1$.

Case 2. Is similar and in fact this is the easy case because the action of $\theta$ on the deviation map is given by $h\left(\theta \sigma_{b}\right)=h\left(\sigma_{b}\right)$. We consider a family of maps $h_{n}: T \rightarrow T$ just as in Case 1 (ii), determined by $\sigma_{a} \mapsto 1, \sigma_{b} \mapsto B^{n} X$, where $X$ denotes the path $\left(b^{q_{1}-q_{2}}, a^{s_{1}-s_{2}}\right)$ deformed into $a^{s_{1}-s_{2}} b^{q_{1}-q_{2}}$ as a path in $a \cup b$.

A Wecken homotopy between $h_{n}$ and $h_{m}$ reduces to the algebraic conclusion that

$$
B^{n} X B^{n} X=\phi B^{m} X B^{m} X \phi^{-1}
$$

A straightforward calculation of $t\left(B^{n} X B^{n} X\right)$ shows that this is impossible when $n \neq m$.

Case 4. In this case we deform $\left(b^{q_{1}-q_{2}} \sigma_{b}, a^{s_{1}-s_{2}}\right)$ into the path $X \sigma_{b}$ contained in $a \cup b$, where $X$ corresponds to the word $a^{k} b^{l}$ with $k=s_{1}-s_{2}, l=q_{1}-q_{2}$. We parametrize in such a way so that $X\left(e^{\pi i}, a^{2 s_{2}}\right)$ deforms into $a \cup b$ by a Wecken homotopy, producing the path $\sigma_{b}^{-1} a^{k} b^{l+1} a^{2 s_{2}}$. That is when $X$ traces out a letter only the $e^{\pi i}$ factor acts on the letter. This action replaces the letter $x$ by $\sigma_{b}^{-1} x \sigma_{b}$ and the path $\sigma_{b}$ by $\sigma_{b}^{-1} b$.

Our family of maps will be $h_{n}$ sending $\sigma_{a} \mapsto 1, \sigma_{b} \mapsto B^{n} X$. Then $h\left(\theta \sigma_{b}\right)=\sigma_{b}^{-1} B^{n} a^{k}$ $\times b^{l+1} a^{2 s_{2}}$.

In this case a Wecken homotopy between the pairs corresponding to $h_{n}$ and $h_{m}$ implies that $B^{n} a^{k} b^{l} B^{n} a^{k} b^{l+1} a^{2 s_{2}}$ and $B^{m} a^{k} b^{l} B^{m} a^{k} b^{l+1} a^{2 s_{2}}$ are conjugate in the free group. The invariant $t()$ can be computed to show that this is only possible when $n=m$.

Case 3. This case is just like Case 4 and the details are left to the reader.

\section{Maps which are not root free}

In Section 2 we studied the 1-parameter root problem for root free maps from an arbitrary closed surface into $K$. We now consider this problem in the presence of roots, and 
to do so we will need to restrict the domain to $K$ as well so that we may take advantage of some known results regarding roots. In this setting being root free (up to homotopy) is equivalent to having absolute degree equal to zero. To see this, [4, Theorem 2] tells us that the Nielsen root number is the same as the absolute degree. (See also [3, Theorem A.3].) By consideration of Theorem 3.3 and Lemma 3.1 in this paper we see that the Nielsen root number is zero exactly when the loop $\alpha$ is mapped to a point. The reader can check that such maps can always be deformed to be root free.

The result we obtain below for roots is the same as that for the torus [13]. We first need the following lemma in the case when the absolute degree is 1.

Lemma 4.1. Let $f: K \rightarrow K$ be a map of absolute degree 1 . Suppose that $y_{1}, \ldots, y_{l}$ is a finite set of points in $K$ such that $f^{-1}\left(y_{i}\right)$ is a single point for each $i$, and further, the local degree of $f$ at $f^{-1}\left(y_{i}\right)$ is independent of $i$. Then we can deform $f$ relative to $f^{-1}\left(y_{1}\right), \ldots, f^{-1}\left(y_{l}\right)$ to a homeomorphism $f^{\prime}$ such that the homotopy $H$ between $f$ and $f^{\prime}$ satisfies \#H( $\left.\cdot, t\right)^{-1}\left(y_{i}\right)=1$ for all $t \in[0,1]$ and $i=1, \ldots, l$.

Proof. For simplicity we assume $i=1$ and set $y=y_{1}$. The proof for $i>1$ is identical. Let $N$ be a small neighborhood of $y$ with $N \backslash y$ foliated by circles $\gamma_{t}, 0<t \leq 1$. Let $M$ be a small neighborhood of $f^{-1}(y)$ with $M \backslash f^{-1}(y)$ foliated by $\delta_{t}$. Since $f^{-1}(y)$ is a single point we can deform $f$ to a map $g$ such that $g^{-1}(y)=f^{-1}(y), g\left(\delta_{t}\right) \subset \gamma_{t}$ for each $t$, and $g(K \backslash M) \subset(K \backslash N)$. As the local degree at $f^{-1}(y)$ is \pm 1 we can assume that $g$ is one-toone on $\delta_{1}$. Moreover, there is such a homotopy between $g$ and $f$ which has a single point in the preimage of $y$ at each level.

Since $g$ has absolute degree 1 , by excision we see that the relative map $g:(K \backslash M, \partial M) \rightarrow$ $(K \backslash N, \partial N)$ also has absolute degree 1. It then follows from [18, Theorem 1.1] that this relative map $g$ is homotopic, rel boundary, to a homeomorphism. Finish by extending to all of $K$ using the constant homotopy from $M$ to $N$ to obtain the desired $f^{\prime}$.

Theorem 4.2. Let $f_{1}, f_{2}: K \rightarrow K$ be homotopic maps each having absolute degree $n$, with $n \neq 0$. If for some $y \in K, \# f_{i}^{-1}(y)=n$ for $i=1,2$, then there is a homotopy $H$ between $f_{1}$ and $f_{2}$ such that $\# H(\cdot, t)^{-1}(y)=n$ for all $t \in[0,1]$.

Proof. We first note that it follows from [4, Theorem 2] that $f_{i \#}\left(\pi_{1}(K)\right)$ is a subgroup of $\pi_{1}(K)$ of index $n$. Let $p: K^{\prime} \rightarrow K$ be the $n$-fold covering which corresponds to the subgroup $f_{i \#}\left(\pi_{1}(K)\right)$ and let $\tilde{f}_{i}: K \rightarrow K^{\prime}$ denote the lift of $f_{i}$. Certainly $\tilde{f}_{i \#}$ is surjective, and since $K^{\prime}$ is either the Torus or the Klein bottle it follows again from [4, Theorem 2] that this map is also injective. Therefore, $\tilde{f}_{i}$ is a homotopy equivalence. So $K^{\prime}$ is a Klein Bottle and $\tilde{f}_{i}$ has absolute degree one. Moreover, $\tilde{f}_{i}$ is homotopic to a homeomorphism and is a Type I map of the form $\alpha \mapsto \alpha, \beta \mapsto \alpha^{s} \beta$.

Let $y_{1}, \ldots, y_{n}$ be the preimage of the point $y$ of the covering map. Then $\tilde{f}_{i \# \text { maps the }}$ set $f_{i}^{-1}(y)$ onto these $n$ points. So, $\tilde{f}_{i}$ viewed as a map on the compliment of these points is also homotopic to a homeomorphism, and we see that the local degree at each point in $f_{i}^{-1}(y)$ has the same value in \pm 1 . By Lemma 4.1 there are homotopies $F_{i}$ with ends $\tilde{f}_{i}$ and a homeomorphism $h_{i}$. Since $h_{1}, h_{2}$ are homotopic, they are also isotopic. Putting together 
$F_{1}, F_{2}$ and the isotopy yields a homotopy $\tilde{H}$ between $\tilde{f}_{1}$ and $\tilde{f}_{2}$ such that the composition $p \circ \tilde{H}$ provides the required homotopy.

\section{The coincidence free case revisited}

In this section we revisit the two stated variations of the 1-parameter coincidence problem. One being the situation where we allow homotopies of both maps and the other being the restricted problem where one map is held constant. The later was addressed in Section 3 of this paper.

The purpose of this section is to show that these two problems are different for coincidences between pairs of maps from the Klein bottle to itself. In general such an analysis is quite difficult. To facilitate calculation we set one of the maps to be the constant map at a point $c$. This will also be the fixed map for the restricted Wecken problem, and as a result this restricted problem reduces to a root problem.

Denote by $e_{1}, e_{2} \in \pi_{1}(K)$ generators which satisfy the relation $e_{1}^{2} e_{2}^{2}=1$. Given a pair of maps $f$ and $g$ which are coincidence free, denote by $(f, g)_{\#}: \pi_{1}(K) \rightarrow \pi_{1}(K \times K-\Delta)$ the homomorphism in the fundamental group induced by the map $(f, g): K \rightarrow K \times K-\Delta$.

Proposition 5.1. Given $f_{1}, f_{2}: K \rightarrow K-c$ consider the two homomorphisms $\left(f_{1}, c\right)_{\#}$ and

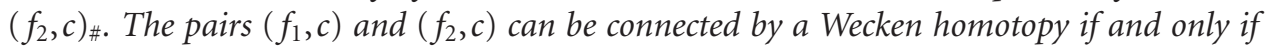
the two homomorphisms are conjugate.

Proof. Assuming the existence of a Wecken homotopy it is well known that the induced homomorphisms in the fundamental group are conjugate (the homotopy is not necessarily base point preserving.) Conversely, since the spaces $K$ and $K \times K-\Delta$ are $K(\pi, 1)$ 's it then follows that the pairs $\left(f_{1}, c\right)$ and $\left(f_{2}, c\right)$ can be joined by a coincidence free homotopy.

By abuse of notation let $e_{1}, e_{2} \in \pi_{1}(K-c)$ denote a basis for the free group where these elements project into $e_{1}, e_{2} \in \pi_{1}(K)$ respectively, and let $B=e_{1}^{2} e_{2}^{2}$.

Corollary 5.2. Let $f_{1}, f_{2}: K \rightarrow K-c$ be two maps such that in the fundamental group they induce the following homomorphisms:

$$
\begin{gathered}
f_{1 \#}\left(e_{1}\right)=e_{1}, \quad f_{1 \#}\left(e_{2}\right)=\left(e_{1}\right)^{-1}, \\
f_{2 \#}\left(e_{1}\right)=e_{1} B^{-1}, \quad f_{2 \#}\left(e_{2}\right)=B\left(e_{1}\right)^{-1} .
\end{gathered}
$$

Then the pairs $\left(f_{1}, c\right)$ and $\left(f_{2}, c\right)$ can be connected by a Wecken homotopy but $f_{1}$ can not be connected to $f_{2}$ by a homotopy which is root free at $c$.

Proof. That $f_{1}$ can not be connected to $f_{2}$ by a root free at $c$ homotopy follows from the fact that $f_{1 \#}\left(e_{1}\right)$ and $f_{2 \#}\left(e_{1}\right)$ are not conjugate as elements of $\pi_{1}(K-c)$. This is straightforward in $K-c$ because the words $e_{1}$ and $e_{1} B^{-1}$ do not have the same exponent sums in the free group $\pi_{1}(K-c)$. To see that $\left(f_{1}, c\right)$ and $\left(f_{2}, c\right)$ can be connected by a Wecken homotopy, by Proposition 5.1 it suffices to see that the two homomorphisms, $\left(f_{1}, c\right)_{\#}$ and $\left(f_{2}, c\right)_{\#}$ are conjugate. Using the relations given in the paper of Scott [17] we have that 
$\rho_{21} \rho_{11} \rho_{21}^{-1}=\rho_{11} B^{-1}$, and so $\rho_{21} \rho_{11}^{-1} \rho_{21}^{-1}=B \rho_{11}^{-1}$. In this notation we have

$$
\begin{array}{cc}
\left(f_{1}, c\right)_{\#}\left(e_{1}\right)=\rho_{11}, \quad\left(f_{1}, c\right)_{\#}\left(e_{2}\right)=\left(\rho_{11}\right)^{-1}, \\
\left(f_{2}, c\right)_{\#}\left(e_{1}\right)=\rho_{11} B^{-1} & \left(f_{2}, c\right)_{\#}\left(e_{2}\right)=B\left(\rho_{11}\right)^{-1} .
\end{array}
$$

This implies that $\rho_{21}\left(f_{1}, c\right)_{\#} \rho_{21}^{-1}=\left(f_{2}, c\right)_{\#}$ and the result follows.

\section{Acknowledgment}

The second author would like to thank the IME at Universidade de" São Paulo for its hospitality during the preparation of this manuscript.

\section{References}

[1] S. Bogatyi, D. L. Gonçalves, and H. Zieschang, The minimal number of roots of surface mappings and quadratic equations in free groups, Mathematische Zeitschrift 236 (2001), no. 3, 419-452.

[2] R. B. S. Brooks, On removing coincidences of two maps when only one, rather than both, of them may be deformed by a homotopy, Pacific Journal of Mathematics 40 (1972), no. 1, 45-52.

[3] R. B. S. Brooks, R. F. Brown, and H. Schirmer, The absolute degree and the Nielsen root number of compositions and Cartesian products of maps, Topology and Its Applications 116 (2001), no. 1, $5-27$.

[4] R. B. S. Brooks and C. Odenthal, Nielsen numbers for roots of maps of aspherical manifolds, Pacific Journal of Mathematics 170 (1995), no. 2, 405-420.

[5] L. E. J. Brouwer, Über die Minimalzahl der Fixpunkte bei den Klassen von eindeutigen stetigen Transformationen der Ringlfächen, Mathematische Annalen 82 (1920), no. 1-2, 94-96.

[6] R. F. Brown and H. Schirmer, Nielsen root theory and Hopf degree theory, Pacific Journal of Mathematics 198 (2001), no. 1, 49-80.

[7] D. Dimovski and R. Geoghegan, One-parameter fixed point theory, Forum Mathematicum 2 (1990), no. 2, 125-154.

[8] R. Dobreńko and J. Jezierski, The coincidence Nielsen number on nonorientable manifolds, Rocky Mountain Journal of Mathematics 23 (1993), no. 1, 67-85.

[9] D. B. A. Epstein, The degree of a map, Proceedings of the London Mathematical Society. Third Series 16 (1966), 369-383.

[10] R. Geoghegan and A. Nicas, Parametrized Lefschetz-Nielsen fixed point theory and Hochschild homology traces, American Journal of Mathematics 116 (1994), no. 2, 397-446.

[11] D. L. Gonçalves, Coincidence of maps between surfaces, Journal of the Korean Mathematical Society 36 (1999), no. 2, 243-256.

[12] D. L. Gonçalves and M. R. Kelly, Maps between surfaces and minimal coincidence sets for homotopies, Topology and Its Applications 116 (2001), no. 1, 91-102.

[13] _ Maps into the torus and minimal coincidence sets for homotopies, Fundamenta Mathematicae 172 (2002), no. 2, 99-106.

[14] J. Jezierski, One codimensional Wecken type theorems, Forum Mathematicum 5 (1993), no. 5, 421-439.

[15] M. R. Kelly, Some examples concerning homotopies of fixed point free maps, Topology and Its Applications 37 (1990), no. 3, 293-297.

[16] H. Schirmer, Fixed point sets of homotopies, Pacific Journal of Mathematics 108 (1983), no. 1, 191-202.

[17] G. P. Scott, Braid groups and the group of homeomorphisms of a surface, Proceedings of the Cambridge Philosophical Society 68 (1970), 605-617. 
[18] R. Skora, The degree of a map between surfaces, Mathematische Annalen 276 (1987), no. 3, 415423.

[19] P. Wong, Coincidences of maps into homogeneous spaces, Manuscripta Mathematica 98 (1999), no. 2, 243-254.

D. L. Gonçalves: Departamento de Matemática, IME-USP, Caixa Postal 66281, Ag. Cidade de São Paulo CEP: 05315-970, São Paulo, SP, Brasil

E-mail address: dlgoncal@ime.usp.br

M. R. Kelly: Department of Mathematics and Computer Science, Loyola University, 6363 St. Charles Avenue, New Orleans, LA 70118, USA

E-mail address: kelly@loyno.edu 